\title{
Selected luminal mucosal complications of adult celiac disease
}

\author{
Hugh J Freeman \\ Department of Medicine \\ (Gastroenterology), University \\ of British Columbia, Vancouver, BC, \\ Canada
}

\begin{abstract}
Celiac disease is a gluten-dependent intestinal disorder that appears to be associated with several clinical conditions. Some involve the luminal mucosa of the stomach and intestinal tract and may, occasionally, complicate the course of celiac disease. Collagenous colitis has been associated with celiac disease and may lead to chronic diarrhea. Conversely, some of these clinical disorders that involve the luminal mucosa of the stomach and intestine may represent the initial clinical presentation of celiac disease. These disorders should be considered in patients with celiac disease who develop recurrent or refractory symptoms despite adherence to a strict gluten-free diet. Detection of collagenous disorders that affect the luminal mucosa of the stomach or intestinal tract may result in recognition of underlying celiac disease.
\end{abstract}

Keywords: celiac disease, collagenous gastritis, collagenous sprue, collagenous colitis, gluten-free diet

Adult celiac disease is a chronic gluten-dependent intestinal disorder that is estimated to affect about $0.5 \%$ to $1 \%$ of the population in Europe and North America, and the disorder is becoming increasingly recognized. ${ }^{1}$ Celiac disease in the adult, even in the very elderly, usually presents with chronic diarrhea and weight loss. ${ }^{2}$ Some may present without diarrhea, but instead with iron deficiency or altered chemistry values (eg, low serum albumin levels). Alternatively, a closely linked disorder may be evident, such as autoimmune thyroid disease, insulin-dependent diabetes, or dermatitis herpetiformis. Finally, positive screening blood test results (eg, tissue transglutaminase antibodies) may lead to eventual diagnosis of celiac disease. In recent years, fewer patients presenting with diarrhea and more patients presenting with positive serology have been noted. ${ }^{2}$

Diagnosis of celiac disease depends on documentation, prior to treatment, of typical changes in proximal small bowel mucosal biopsies. These have been previously detailed $^{1}$ and include: absent or rudimentary villi, increased lamina propria lymphoid cell elements (ie, plasma cells, lymphocytes), increased intra-epithelial lymphocytes and crypt epithelial cell hyperplasia. A second critical step in documentation of celiac disease is definition of an unequivocal clinical and/or pathological response to a gluten-free diet. In adults, resolution of diarrhea, weight gain and normalization of abnormal blood and chemistry values on a gluten-free diet is usually sufficient to provide a convincing clinical diagnosis. However, if limited symptoms were initially evident, then additional biopsies may be needed to show histological improvement. With a gluten-free diet, the mucosa reverts towards normal, although prolonged periods of dietary modification may be needed, particularly in older adults. ${ }^{3}$

Celiac disease causes changes along the length of the small intestine. The extent of this change correlates better with clinical status than the severity of the biopsy changes in the proximal small bowel. In classic disease with malabsorption, severe changes 
may extend well into the jejunum. Less severe mucosal alterations are detected in the ileum, even though the ileal mucosa has been shown to be highly susceptible to gluten (infused through long intestinal tubes). ${ }^{4}$ It is possible that this proximal-to-distal gradient in the severity of pathological changes reflects the higher concentrations of dietary gluten (or its derivative peptides) in the proximal small intestine. Alternatively, changes may be indirect, resulting from immune-mediated effects of circulating memory T-cells. ${ }^{5}$ With extensive involvement of the small intestine, diarrhea and malabsorption of many nutrients occur. With less involvement, deficits may be limited, and diarrhea or weight loss may not be evident. Moreover, resolution of abnormal histological changes tends to occur initially in the distal small intestine, and later in the more proximal sites.

In celiac disease, other superimposed luminal mucosal disorders may occur in the stomach or the intestinal tract. Usually, most develop with established celiac disease, but these may also represent the presenting clinical feature of celiac disease in the adult. In celiac disease with recurrent or refractory symptoms, a number of possible causes require consideration. Most often, poor compliance or intentional lack of compliance to a gluten-free diet or ingestion of a source of unrecognized gluten are the most common causes. But other associated disorders, including pancreatic exocrine failure, ${ }^{6}$ or neoplastic disorders, particularly lymphoproliferative and other intestinal malignancies ${ }^{7}$ may be important. Persistent or recurrent symptoms may also be due to other causes, including the collagenous mucosal inflammatory disorders. ${ }^{8}$ Some are rarely encountered, and as a group, may have a limited prevalence in some centers, ${ }^{9}$ however, increased awareness of these conditions by specialist clinicians may lead to increased recognition in adult celiac disease.

\section{Collagenous sprue}

In 1970, a 51-yr-old female with apparent celiac disease was recorded with refractory malabsorption. ${ }^{10}$ An unusual histological abnormality in the small intestine was described consisting of an eosinophilic sub-epithelial hyaline-like material in the lamina propria. The deposits had histochemical staining characteristics of collagen, and ultra-structural studies confirmed the presence of an electron-dense material with the characteristic 640. A axial periodicity of collagen fibers. Her subsequent clinical course was characterized by worsening malabsorption, diarrhea and weight loss. Steroids caused temporary resolution of her diarrhea. Later reports described severe and long-standing malabsorption with diarrhea and progressive weight loss, flattened small intestinal villous architecture, and these same distinctive sub-epithelial collagen deposits.

A relationship between collagenous sprue and celiac disease was controversial. Some felt that the collagen deposits simply represented a prognostic marker of a poor outcome in celiac disease. ${ }^{11}$ Others considered the disorder to be an entirely new, previously unrecognized, entity poorly responsive to a gluten-free diet. ${ }^{12}$ Both entities share many common elements. Hyposplenism and positive endomysial antibodies have been detected in both disorders. ${ }^{13}$ Moreover, some complications ordinarily associated with celiac disease have been associated with collagneous sprue. Small intestinal ulceration and perforation may develop. ${ }^{14}$ Atypical immunohistochemical changes with gene rearrangement defined by polymerase chain reaction reported in refractory sprue (or sprue-like intestinal disease) have been noted in collagenous sprue. ${ }^{14,15}$ Malignant lymphoma may develop during the clinical course of collagenous sprue. ${ }^{14,16,17}$ As in celiac disease, both T-cell lymphoma ${ }^{16}$ and B-cell lymphoma ${ }^{17}$ have been recorded in collagenous sprue.

The natural history of collagenous sprue has been characterized by continued malabsorption, usually of multiple nutrients, and a lethal outcome. Some recent reports, however, with extensive biopsy studies have also noted complete disappearance of these abnormal collagen deposits after treatment with steroids for periods of up to almost four years. ${ }^{18}$ Thus, the lesion may be reversible, at least temporarily, for prolonged periods. It may also be that the collagen deposits have different causes, in some instances being steroid-responsive. Of note, in a recent report, collagen deposits completely revolved after resection of a localized colon cancer, suggesting a possible paraneoplastic phenomenon, expressed as this histopathologic marker in the intestinal mucosa. ${ }^{19}$

\section{Collagenous colitis}

In 1976, similar deposits were detected in the colon in patients with a watery diarrhea syndrome. ${ }^{20,21}$ Colorectal biopsies showed lamina propria sub-epithelial hyaline deposits similar to the deposits reported in the small intestine with collagenous sprue. Although the endoscopic appearances were normal or near-normal, a microscopic mucosal inflammatory process was also present with increased lymphocytes and plasma cells. Intra-epithelial lymphocytes were also increased. The hyaline deposits stained positively with trichrome, characteristic of collagen and ultrastructural studies confirmed the presence of collagen fibers.

Later reports from large registries further detailed the clinical features. ${ }^{22,23}$ Most patients were middle-aged or 
elderly females, however, collagenous colitis has also been detected in males and children. ${ }^{24}$ Diarrhea was usually watery, nonbloody, and sometimes nocturnal, often with intermittent abdominal pain and weight loss. Often, the diarrhea was present for weeks or months, resolving spontaneously for variable periods of time, then recurring with remissions and relapses over many years.

The pathogenesis of the diarrhea in collagenous colitis has been evaluated but still needs to be elucidated. Some functional studies of the small bowel and pancreas were normal. ${ }^{25}$ Interestingly, however, some early case studies both adult and childhood celiac disease in collagenous colitis. ${ }^{26-28}$ Serological investigations failed to show a linkage with celiac disease ${ }^{29}$ and two retrospective studies of small bowel biopsies in collagenous colitis were contradictory. ${ }^{30,31}$ However, a long-term prospective small bowel biopsy study in collagenous colitis documented celiac disease in over $20 \%$ of subjects. ${ }^{32}$ Recognition of celiac disease may be important from a therapeutic perspective because a gluten-free diet may resolve the diarrhea without need for other pharmacologic treatment. In addition, other autoimmune disorders such as thyroiditis may be seen in collagenous colitis at similar frequencies to that recorded in celiac disease. ${ }^{32}$ Finally, splenic hypofunction, often seen in celiac disease, has been recorded in collagenous colitis. ${ }^{33}$

Some have classified collagenous colitis as a form of microscopic colitis. In part, this is due to a clinical paradigm that differentiates this entity from other inflammatory bowel disease disorders (eg, ulcerative colitis, Crohn's disease) with macroscopically visible changes detected during endoscopic evaluation. Another type of microscopic colitis is lymphocytic colitis that serves to emphasize the predominant cell type in cases of watery diarrhea with macroscopically normal mucosa. Some believe that there may be an hypothetical relationship between lymphocytic colitis and collagenous colitis, however, the absence of collagen deposits (in lymphocytic colitis) may simply reflect their patchy and focal distribution in collagenous colitis. Interestingly, though, increased epithelial lymphocytosis (without collagen deposits) has been defined in colorectal mucosal biopsies from patients with celiac disease. ${ }^{34}$ Bloody diarrhea is very unusual in collagenous colitis and usually suggests that another disorder is present and, recently, evolution of collagenous colitis into severe and extensive ulcerative colitis has been documented. ${ }^{35}$

Complications of collagenous colitis may also occur, and these may be seen in adult celiac disease with concomitant collagenous colitis. Surface epithelial sloughing is commonly seen in colonic biopsies leaving a "naked" sub-epithelial collagen deposit. In some, altered mucosal permeability has been recorded and protein-losing enteropathy has been noted in the absence of small intestinal disease. ${ }^{8}$ Submucosal "dissection" has been described. ${ }^{36}$ Colonic fracturing after endoscopic instrumentation, possibly related to air insufflation and barotraumas, or insertion of barium contrast agents, has been recorded. ${ }^{37}$ Recently, a report entitled "cat scratch colon" emphasized the macroscopic changes in the colon in collagenous colitis. ${ }^{38}$ Spontaneous peritonitis with colonic perforation has also been recorded. ${ }^{39}$ A rarity of malignant disease has been noted in collagenous colitis, such as colonic carcinoma. ${ }^{40}$ Other neoplasms have also been recorded including lymphoproliferative disorders ${ }^{41}$ and carcinoids. ${ }^{42}$

\section{Gastric inflammatory disorders}

Although lymphocytic gastritis was originally noted in patients with celiac disease or sprue-like intestinal disease, ${ }^{43}$ other chronic gastritis types may occur. In 1989, a form of chronic gastritis was initially described characterized by gastric sub-epithelial collagen deposits with a background inflammatory process. ${ }^{44}$ The presence of collagen in these deposits was confirmed using ultrastructural methods and observed in both adults and children. ${ }^{45}$ Later, this entity was noted in celiac disease. ${ }^{46}$ Some recent studies have shown concomitant collagen deposits elsewhere in the intestinal mucosa, suggesting that, in some instances, collagenous disease may be a far more extensive inflammatory disease process. $^{47}$

\section{Conclusion}

Diagnosis of celiac disease is usually straightforward, but occasionally, it may prove to be difficult. Once established, however, the clinician should be alert to other luminal mucosal disorders, such as collagenous colitis, that may complicate the clinical course of celiac disease. Conversely, collagenous colitis or other specific luminal mucosal disorders may be the presenting manifestation of underlying celiac disease.

\section{Disclosure}

The author reports no conflicts of interest in this work.

\section{References}

1. Freeman HJ. Pearls and pitfalls in the diagnosis of adult celiac disease. Can J Gastroenterol. 2008;22:273-280.

2. Lo W, Sano K, Lebwohl B, Diamond B, Green PH. Changing presentation of adult celiac disease. Dig Dis Sci. 2003;48:395-398.

3. Tursi A, Brandimarte G, Giorgetti GM, et al. Endoscopic and histologic findings in the duodenum of adults with celiac disease before and after changing to a gluten-free diet: a 2-year prospective study. Endoscopy. 2006;38:702-707. 
4. MacDonald WC, Brandborg LL, Flick AK, Trier JS, Rubin CE. Studies of celiac sprue. IV. The response of the whole length of the small bowel to a gluten-free diet. Gastroenterology. 1964;47:573-589.

5. MacDonald TT. T-cell mediated intestinal injury. In: Marsh MN, editor. Celiac Disease. London: Blackwell Scientific Publications; 1992. p. 283-304.

6. Freeman HJ. Pancreatic endocrine and exocrine changes in celiac disease. World J Gastroenterol. 2007;13:6344-6346.

7. Freeman HJ. Lymphoproliferative and intestinal malignancies in 214 patients with biopsy-defined celiac disease. J Clin Gastroenterol. 2004;38:429-434.

8. Freeman HJ. Collagenous mucosal inflammatory diseases of the gastrointestinal tract. Gastroenterology. 2005;129:338-350.

9. Fernandez-Banares F, Esteve M, Farre C, et al. Predisposing HLADQ2 and HLA-DQ8 haplotypes of celiac disease and associated enteropathy in microscopic colitis. Eur J Gastroenterol Hepatol. 2005;17:1333-1338.

10. Weinstein WM, Saunders DR, Tytgat GN, Rubin CE. Collagenous sprue - an unrecognized type of malabsorption. $N$ Engl J Med. 1970;283:129-1301.

11. Bossart R, Henry K, Booth CC, Doe WF. Subepithelial collagen in intestinal malabsorption. Gut. 1975;16:18-22.

12. Perera DR, Weinstein WM, Rubin CE. Small intestinal biopsy. Hum Pathol. 1975;6:157-217.

13. Freeman HJ. Hyposplenism, antiendomysial antibodies and lymphocytic colitis in collagenous sprue. Can J Gastroenterol. 1999;13:347-350

14. Medlicott SA, Beck PL, Loken S, Crabtree T. Synchronous collagenous sprue and enteropathy-associated T-cell lymphoma. Lancet. 2000;356:203-208

15. Cellier C, Dellabesse E, Helmer C, et al. Refractory sprue, celiac disease, and enteropathy-associated T-cell lymphoma. Lancet. 2000;356:203-208

16. Freeman HJ. Collagenous sprue associated with an extensive T-cell lymphoma. J Clin Gastroenterol. 2003;17:111-113.

17. Robert ME, Ament ME, Weinstein WM. The histologic spectrum and clinical outcome of refractory and unclassified sprue. Am J Surg Pathol. 2000;24:676-687.

18. Freeman HJ, Davis JE, Myers DM. Complete histological resolution of collagenous sprue. Can J Gastroenterol. 2004;18:333-336.

19. Freeman HJ, Berean KW. Resolution of paraneoplastic collagenous enterocolitis after resection of colon cancer. Can J Gastroenterol. 2006;20:357-360.

20. Freeman HJ, Weinstein WM, Shnitka TK, Wensel RH, Sartor VE. Watery diarrhea syndrome associated with a lesion of the colonic basement membrane-lamina propria interface. Ann Roy Coll Phys Surg Can. 1976;9:45.

21. Lindstrom CG. Collagenous colitis with watery diarrhea - a new entity? Pathol Eur. 1976;11:87-89.

22. Olesen M, Eriksson S, Bohr J, Jarnerot G, Tysk C. Microscopic colitis: a common diarrheal disease. An epidemiological study in Orebro, Sweden, 1993-1998. Gut. 2004;53:346-350.

23. Agnarsdottir M, Gunnlaugsson O, Orvar KB, et al. Collagenous and lymphocytic colitis in Iceland. Dig Dis. 2002;47:1122-1128.

24. Camarero C, Leon F, Colino E, et al. Collagenous colitis in children: clinicopathologic, microbiologic, and immunologic factors. J Pediatr Gastroenterol Nutr. 2003;37:508-513.

25. Bo-Lim GW, Vendrell DD, Lee E, Fordtran JS. An evaluation of the significance of microscopic colitis in patients with chronic diarrhea. J Clin Invest. 1985;75:1559-1569.
26. Hamilton I, Sanders S, Hopwood D, Bouchier IA. Collagenous colitis associated with small intestinal villous atrophy. Gut. 1986;27:1394-1398.

27. O'Mahoney S, Nawroz IM, Ferguson A. Coeliac disease and collagenous colitis. Postgrad Med J. 1990;66:238-241.

28. McCashland TM, Donovan JP, Strobach RS, Linder J, Quigley EM. Collagenous enterocolitis: a manifestation of gluten-sensitive enteropathy. J Clin Gasroenterol. 1992;15:45-51.

29. Gillett HR, Freeman HJ. Prevalence of celiac disease in collagenous and lymphocytic colitis. Can J Gastroenterol. 2000;14:919-921.

30. Armes J, Gee DC, Macrae FA, Schroeder W, Bhathal PS. Collagenous colitis: jejunal and colorectal pathology. J Clin Pathol. 1992;45:784-787.

31. Zins BJ, Tremaine WJ, Carpenter HA. Collagenous colitis: mucosal biopsies and association with fecal leukocytes. Mayo Clin Proc. 1995; 70:430-433.

32. Freeman HJ. Collagenous colitis as the presenting feature of biopsydefined celiac disease. J Clin Gastroenterol. 2004;38:664-668.

33. Freeman HJ. Functional asplenia and microscopic (collagenous) colitis. Can J Gastroenterol. 1996;10:443-446.

34. Wolber R, Owen D, Freeman HJ. Colonic lymphocytosis in patients with celiac sprue. Hum Pathol. 1990;21:1092-1096.

35. Freeman HJ, Berean KW, Nimmo M. Evolution of collagenous colitis into severe and extensive ulcerative colitis. Can J Gastroenterol. 2007:21:315-318.

36. Mitchell JD, Teague R, Bolton R, Lowes J. Submucosal "dissection" in collagenous colitis. Gut. 2004;53:470.

37. Sherman A, Ackert JJ, Rajapaksa R, West AB, Oweity T. Fractured colon: an endoscopically distinctive lesion associated with colonic perforation following colonoscopy in patients with collagenous colitis. J Clin Gastroenterol. 2004;38:341-345.

38. McDonnell WM, Loura F, Pointon MJ, Greenson JK. Cat scratch colon. Endoscopy. 2007;39:459-461.

39. Freeman HJ, James D, Mahoney CJ. Spontaneous peritonitis from perforation of the colon in collagenous colitis. Can J Gastroenterol. 2001;15:265-267.

40. Chan JL, Tersmette AC, Offerhaus GJ, Gruber SB, Bayless TM, Giardiello FM. Cancer risk in collagenous colitis. Inflamm Bowel Dis. 1999;5:40-43.

41. Freeman HJ. Lymphoproliferative disorders in collagenous colitis. Inflamm Bowel Dis. 2005;11:781-782.

42. Nussinson E, Samara M, Vigder L, Shafer I, Tzur N. Concurrent collagenous colitis and multiple ileal carcinoids. Dig Dis Sci. 1988;33:1040-1044.

43. Wolber R, Owen D, DelBuono L, Appelman H, Freeman HJ. Lymphocytic gastritis in patients with celiac sprue or sprue-like intestinal disease. Gastroenterology. 1990;98:310-315.

44. Freeman HJ, Piercey JR, Raine RJ. Collagenous gastritis. Can J Gastroenterol. 1989;3:171-174.

45. Colletti RB, Trainer TD. Collagenous gastritis. Gastroenterology. 1989;97:1552-1555.

46. Stancu M, De Petris G, Palumbo TP, Lev R. Collagenous gastritis associated with lymphocytic gastritis and celiac disease. Arch Pathol Lab Med. 2001;125:1579-1584.

47. Freeman HJ. Topographic mapping of collagenous gastritis. Can J Gastroenterol. 2001;15:475-478. 